PHARMACOEPIDEMIOLOGY AND DRUG SAFETY 2011; 20: 1225-1236

Published online 3 October 2011 in Wiley Online Library (wileyonlinelibrary.com) DOI: 10.1002/pds.2227

REVIEW

\title{
Stroke risk and NSAIDs: a systematic review of observational studies ${ }^{\dagger}$
}

\author{
Cristina Varas-Lorenzo ${ }^{1 *}$, Nuria Riera-Guardia ${ }^{1}$, Brian Calingaert ${ }^{2}$, Jordi Castellsague ${ }^{1}$, Antoine Pariente ${ }^{3}$, \\ Lorenza Scotti ${ }^{4}$, Miriam Sturkenboom ${ }^{5}$ and Susana Perez-Gutthann ${ }^{1}$ \\ ${ }^{1}$ RTI Health Solutions, Barcelona, Spain \\ ${ }^{2}$ RTI Health Solutions, Research Triangle Park, NC, USA \\ ${ }^{3}$ Universite Victor Segalen, Bordeux, France \\ ${ }^{4}$ Department of Statistics, Unit of Biostatistics and Epidemiology, University of Milano-Bicocca, Milan, Italy \\ ${ }^{5}$ Erasmus University Medical Center, Rotterdam, Netherlands
}

\begin{abstract}
Aims To perform a quantitative systematic review of observational studies on the risk of stroke associated with the use of individual NSAIDs. Methods and results Searches were conducted using the Medline database within PubMed (1990-2008). Observational cohort or casecontrol studies were eligible if reported on the risk of cardiovascular events associated with individual NSAIDs versus the nonuse of NSAIDs. We found 3193 articles, in which 75 were eligible for review and abstraction. Of the 75 articles, 6 reported relative risk (RR) of stroke. Data were abstracted into a database using a standardized entry form. Two authors assessed study quality, and discrepancies were resolved by consensus.

The pooled RR of all subtypes of incident stroke was increased with the current use of rofecoxib $(\mathrm{RR}=1.64,95 \% \mathrm{CI}=1.15-2.33)$ and diclofenac $(\mathrm{RR}=1.27,95 \% \mathrm{CI}=1.08-1.48)$. The pooled estimates for naproxen, ibuprofen, and celecoxib were close to unity. The risk of ischemic stroke was also increased with rofecoxib $(\mathrm{RR}=1.82,95 \% \mathrm{CI}=1.09-3.04)$ and diclofenac $(\mathrm{RR}=1.20,95 \% \mathrm{CI}=0.99-1.45)$. Data were inadequate to estimate the pooled RR by dose and duration, for other individual NSAIDs or nonischemic stroke subtypes.

Conclusion This meta-analysis supports an increased risk of ischemic stroke with the current use of rofecoxib and diclofenac. Additional studies are required to evaluate most individual NSAIDS, the effect of dose and duration, and the subtypes of stroke. Copyright (C) 2011 John Wiley \& Sons, Ltd.
\end{abstract}

KEY WORDS—stroke; meta-analysis; observational studies; anti-inflammatory agents; nonsteroidal; epidemiology

Received 25 February 2011; Revised 13 May 2011; Accepted 12 July 2011

\section{INTRODUCTION}

The cardiovascular safety of NSAIDs is still under scrutiny. The introduction of selective cyclooxygenase $2(\mathrm{COX}-2)$ inhibitors raised concern after patients with rheumatoid arthritis randomized to rofecoxib experienced a greater risk of acute myocardial infarction (AMI) over those randomized to naproxen. ${ }^{1}$ A voluntary

\footnotetext{
*Correspondence to: C. Varas-Lorenzo, RTI Health Solutions, Travesera de
} Gracia 56, Atico 1º, 08006, Barcelona 02451, Spain. E-mail: cvaras@ rti.org

\footnotetext{
${ }^{\dagger} \mathrm{NRG}$ and CVL abstracted and compiled the data, BC performed the analyses, and CVL drafted the manuscript. CVL, NRG, BC, JC, AP, LS, MS, and SPG facilitated the interpretation of the findings. CVL, NRG, JC, AP, LS, MS, and SPG oversaw the design of the study and helped to draft the manuscript. All coauthors participated in the writing of the manuscript and approved the version submitted for publication.

On behalf of the Investigators of The Safety of Nonsteroidal Anti-inflammatory Drugs (SOS) project http://www.sos-nsaids-project.org/
}

withdrawal of rofecoxib worldwide followed the results of the Adenomatous Polyp Prevention on Vioxx trial showing an increased risk of cardiovascular events for rofecoxib over placebo. ${ }^{2}$ A dose-related increased risk of cardiovascular events was also observed in randomized clinical trials performed with celecoxib, ${ }^{3}$ valdecoxib, ${ }^{4}$ and parecoxib. ${ }^{4}$ The Food and Drug Administration stopped the Alzheimer's Disease Anti-inflammatory Prevention Trial because of an increase in cardiovascular events in naproxen as compared with placebo. ${ }^{5}$ The review of selective COX-2 inhibitors safety by the Food and Drug Administration and the European Medicines Agency resulted in their contraindication in patients with ischemic heart disease, stroke, or peripheral arterial disease. The cardiovascular safety of traditional NSAIDs was also of concern.

The syntheses of published interventional and observational studies conclude that both selective and 
nonselective COX-2 inhibitors increase the risk of serious cardiovascular events, and this risk varies across individual NSAIDs. ${ }^{6-9}$ Results from observational studies are consistent with those from interventional studies regarding the effects of individual NSAIDs on the risk of AMI. Cardiovascular toxicity associated with selective COX-2 and some traditional NSAIDs is mediated through a common mechanism involving the inhibition of COX-2-dependent prostacyclin. Most of NSAIDs are functionally selective for COX-2 at therapeutic doses. ${ }^{10}$ With the exception of aspirin, the only NSAID that lacks functional COX-2 selectivity in platelets is naproxen, and this is seen only at high doses in some individuals. ${ }^{11}$ Stroke is one of the leading causes of morbidity and mortality worldwide. Any potential increase in the risk of stroke, although low, associated with the use of NSAIDs will have an important health effect at the population level. However, there is limited evidence from interventional studies on the effect of individual NSAIDs on the risk of stroke. We synthesized the evidence from observational studies to assess the potential differential effects of individual NSAIDs on the risk of stroke.

The Safety of Nonsteroidal Anti-inflammatory Drugs (SOS) project is a research and development project funded by the Health Area of the European Commission under the VII Framework Programme. One of the first steps of this project was a quantitative systematic literature review of observational studies to assess the risk of cardiovascular events associated with the use of NSAIDs for planning future research. We summarized first the estimates of the effect of individual NSAIDs on the risk of stroke from published observational studies. We followed the PRISMA guidelines for reports of systematic reviews (http//www. prisma-statement.org/).

\section{METHODS}

\section{Literature search}

We performed a systematic literature search on cardiovascular events in the Medline database within PubMed using free text search terms and medical subject headings for stroke, myocardial infarction, heart failure, left ventricular dysfunction, acute coronary syndrome, sudden cardiac death, and nonsteroidal anti-inflammatory agents. We examined references of relevant articles for additional sources.

Eligible studies for review were observational cohort or case-control studies published in English in peer-reviewed journals between January 1, 1990, and November 30, 2008. The initial broad search identified
3203 articles, but 3128 articles did not meet the inclusion criteria for study design or study medications. The main reasons for exclusion were partial study reports (letters, comments, and abstracts), noncomparative observational studies, and studies evaluating inappropriate end points or exposures. The review of the remaining 75 studies led to the exclusion of 20 studies, and 55 articles reported results on the use of individual NSAIDs and cardiovascular end points (acute coronary syndrome, 36; stroke, $8^{12-19}$; heart failure, 6; death, 2; and composite end point, 8). The details of article identification and selection processes are in Figure 1.

Studies for inclusion in the present meta-analysis were required to provide measures of association comparing the risk of acute ischemic and/or hemorrhagic stroke between the users of individual NSAIDs and the nonusers. Two studies ${ }^{16,17}$ were excluded because they used other reference categories. Data were abstracted into a database using a standardized entry form.

\section{Assessment of study quality}

We used the Newcastle-Ottawa Scale $(\mathrm{NOS})^{20}$ to evaluate study quality components for cohort or case-control studies, as recommended by the Cochrane Non-Randomized Studies Methods Working Group. The NOS quality scoring focuses on the selection of study groups, the comparability between the study groups, and the ascertainment of the exposure in case-control studies or the outcome in cohort studies. Two investigators (NR and CV) performed the quality evaluation, and discordances were solved by consensus.

\section{Statistical analysis}

We pooled the ORs or relative risks (RR), for each individual NSAID estimated in each study. According to the data available in each study, we conducted pooled prespecified analyses for incident and recurrent cases combined, incident cases only, prevalent and new users of each individual NSAID combined, and new users only. We estimated pooled RRs separately for overall stroke and ischemic stroke. Data were insufficient to estimate the effect of dose and duration for individual NSAIDs.

We estimated pooled RRs and 95\% CI for the effect of each NSAID with at least three independent point estimates available by weighting study estimates by the inverse of the variance and estimating linear predictors for the log effect measure. ${ }^{21}$ Fixed and random effects were calculated. Forest plots were constructed on the basis of the pooled RR estimated from the random effects models. Heterogeneity between studies 


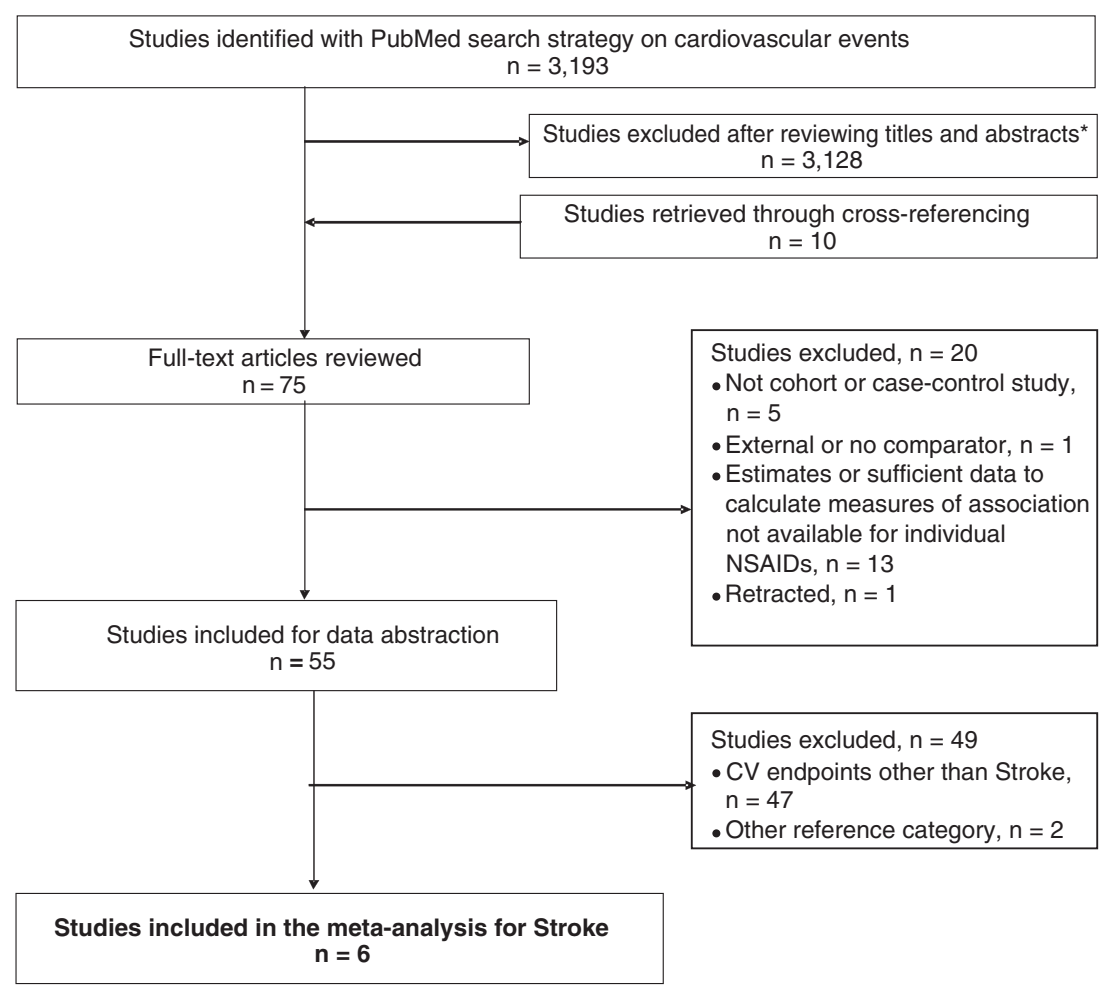

Figure 1. Flow chart of identification and selection of studies; *main reasons for exclusions included the following: a) Not a cohort or a case-control study; b) No fully study report; c) Non-comparative studies or studies with external comparisons groups; d) Estimates or sufficient data to calculate measures of association were not available for individual NSAIDs; e) Studies conducted in hospitalized or institutionalized patients; f) Inappropriate endpoint

was assessed by graphical inspections of the forest plots and by Cochran's $\chi^{2}$ test of homogeneity and $\mathrm{Tau}^{2}$ for random effect models. The Higgins $I^{2}$ statistic was used to describe the percentage of betweenstudy variability in effect estimates that is attributable to true heterogeneity rather than chance. Publication bias was examined by visual evaluation of funnel plots. The analysis was conducted using Review Manager (RevMan) (Version 5.0.22 Copenhagen; The Nordic Cochrane Centre, The Cochrane Collaboration, 2009).

\section{RESULTS}

\section{Characteristics and quality of included studies}

Table 1 describes the main features of the six included studies in the meta-analysis, conducted in selected US and European populations. ${ }^{12-15,18,19}$ Four studies were cohort studies; two were nested case-control studies. Five studies used data collected in electronic databases. One prospective cohort study of elderly was supplemented by electronic drug dispensing and hospital discharge data. Two studies used both outpatient and hospital diagnoses. ${ }^{13,15}$ Two studies were restricted to ischemic stroke ${ }^{13,19}$; four studies also included hemorrhagic stroke, including or excluding subarachnoid hemorrhage (SAH). Overall, four studies provided estimates for ischemic stroke. ${ }^{13-15,19}$ Four studies were restricted to the evaluation of incident events.

Naproxen, ibuprofen, diclofenac, celecoxib, and rofecoxib were the most frequently evaluated. The current use of the individual NSAID was defined as the use at the index date or the use during a variable time window before the index date. Information on dose, duration, and aspirin use was scarce in these studies.

The studies had good quality: four studies scored the maximum NOS score for selection of subjects. ${ }^{13-15,18}$ All studies scored the maximum for comparability and for the ascertainment of outcome (cohort studies) or exposure (case-control studies).

\section{Meta-analysis}

Table 2 shows the results of the pooled analysis for all types of stroke and for the studies of incident strokes. Heterogeneity between studies was present for all individual NSAIDs except diclofenac and was reduced in 
C. VARAS-LORENZO ET AL.

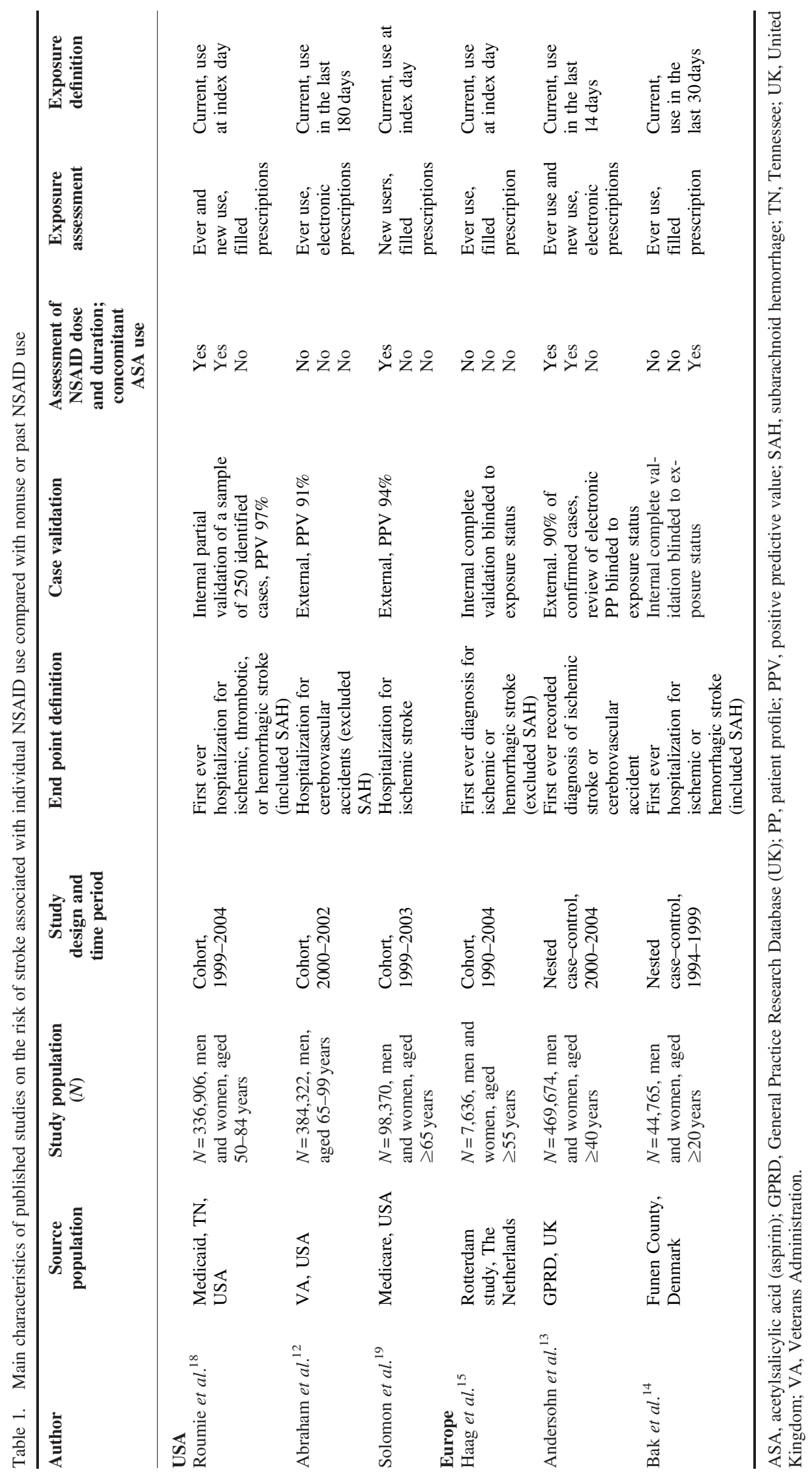


Table 2. RR of stroke associated with naproxen, ibuprofen, diclofenac, celecoxib, and rofecoxib use compared with no NSAID use

\begin{tabular}{|c|c|c|c|c|c|c|}
\hline Author (no. studies) & Cases $(N)$ & $\begin{array}{l}\text { Naproxen RR } \\
(95 \% \text { CI) }\end{array}$ & $\begin{array}{c}\text { Ibuprofen RR } \\
\text { (95\% CI) }\end{array}$ & $\begin{array}{c}\text { Diclofenac RR } \\
\text { (95\% CI) }\end{array}$ & $\begin{array}{l}\text { Celecoxib RR } \\
\text { (95\% CI) }\end{array}$ & $\begin{array}{l}\text { Rofecoxib RR } \\
\text { (95\% CI) }\end{array}$ \\
\hline \multicolumn{7}{|l|}{$\begin{array}{l}\text { All types of stroke } \\
\qquad(n=6)^{*}\end{array}$} \\
\hline Abraham et al. ${ }^{12}$ & NR & $2.00(1.49-2.70)$ & $1.70(1.24-2.32)$ & NA & $1.70(1.14-2.54)$ & $3.00(2.04-4.42)$ \\
\hline Andersohn et al. ${ }^{13 \dagger}$ & 684 & $1.16(0.80-1.70)$ & $1.12(0.91-1.37)$ & $1.32(1.10-1.57)$ & $1.07(0.79-1.44)$ & $1.71(1.33-2.18)$ \\
\hline Bak et al. ${ }^{14 \dagger}$ & 158 & $0.70(0.44-1.13)$ & $1.30(1.03-1.64)$ & $1.10(0.70-1.70)$ & NA & NA \\
\hline Haag et al. ${ }^{15 \dagger}$ & 52 & $2.63(1.47-4.72)$ & $1.47(0.73-3.00)$ & $1.60(1.00-2.57)$ & NA & $3.38(1.48-7.74)$ \\
\hline Roumie et al. $^{18^{\dagger}}$ & 574 & $0.94(0.80-1.11)$ & $0.88(0.73-1.06)$ & $0.94(0.59-1.49)$ & $1.04(0.87-1.23)$ & $1.28(1.06-1.53)$ \\
\hline \multirow[t]{2}{*}{ Solomon et al. ${ }^{19}$} & 1904 & $0.83(0.67-1.04)$ & $0.95(0.78-1.16)$ & $0.98(0.75-1.29)$ & $1.00(0.92-1.09)$ & $1.15(1.04-1.26)$ \\
\hline & & & & Pooled RR $(95 \%$ CI $)$ & & \\
\hline Random effects & & $1.19(0.85-1.65)$ & $1.15(0.95-1.39)$ & $1.17(0.98-1.40)$ & $1.08(0.93-1.25)$ & $1.70(1.25-2.31)$ \\
\hline Fixed effects & & $1.05(0.94-1.17)$ & $1.08(0.98-1.19)$ & $1.20(1.05-1.36)$ & $1.03(0.96-1.10)$ & $1.27(1.18-1.38)$ \\
\hline Heterogeneity $(P)$ & & $<0.00001$ & 0.004 & 0.21 & 0.09 & $<0.00001$ \\
\hline \multicolumn{7}{|l|}{ Incident stroke $(n=4)^{\dagger}$} \\
\hline & & & & Pooled RR (95\% CI) & & \\
\hline Random effects & & $1.14(0.76-1.69)$ & $1.10(0.89-1.36)$ & $1.27(1.08-1.48)$ & $1.04(0.90-1.21)$ & $1.64(1.15-2.33)$ \\
\hline Fixed effects & & $1.00(0.87-1.15)$ & $1.06(0.95-1.20)$ & $1.27(1.10-1.47)$ & $1.04(0.90,1.21)$ & $1.45(1.26-1.68)$ \\
\hline Heterogeneity $(P)$ & & 0.003 & 0.05 & 0.37 & 0.85 & 0.02 \\
\hline
\end{tabular}

Results from published studies and pooled estimates, overall analysis, and studies of incident stroke.

NR, not reported; NA, not applicable.

*All types of stroke, incident plus prevalent cases of all subtypes of stroke.

${ }^{\dagger}$ Denotes studies restricted to incident events.

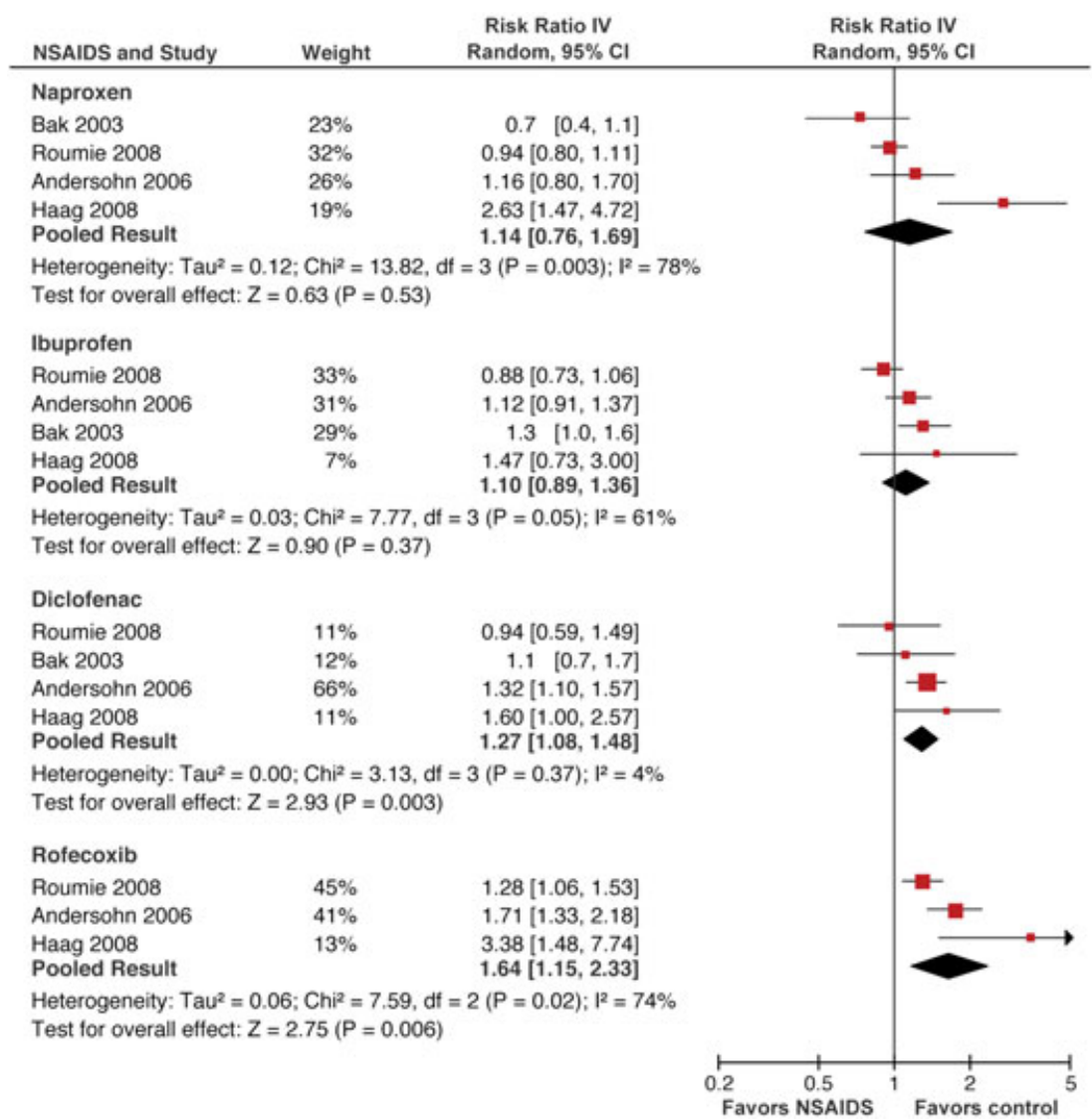

Figure 2. Forest plots of the risk of incident stroke, ischemic and hemorrhagic, associated with current use of naproxen, ibuprofen, diclofenac and rofecoxib relative to nonuse; results from published studies $(n=4)$ and pooled estimates by random effects 
the analysis of incident stroke. Figure 2 displays individual study results and pooled estimates for each individual NSAID on the risk of incident stroke. The pooled RRs were 1.64 (95\% CI $=1.15-2.33)$ for rofecoxib and 1.27 (95\% $\mathrm{CI}=1.08-1.48)$ for diclofenac. The pooled estimates for naproxen, ibuprofen, and celecoxib were close to unity. Estimates for celecoxib were based on only two studies (Table 2).

The apex in the funnel plot for all types of stroke is pointing up around a RR of 1.3 (Figure 3). Higher estimates for naproxen and rofecoxib were observed in the smallest study. ${ }^{15}$ No small studies reporting a reduction on the risk of stroke were published, which might indicate some publication bias.

\section{Pooled RR estimates by stroke subtype}

Figure 4 shows the forest plots of the pooled estimates of studies reporting the risk of ischemic stroke associated using individual NSAIDs compared with the nonuse of NSAIDs. The pooled RRs were $1.82(95 \% \mathrm{CI}=1.09-3.04)$ for rofecoxib and 1.20 (95\% CI $=0.99-1.45)$ for diclofenac, similar to those observed in the analysis of all stroke types combined. Only one study evaluated separately the risk of hemorrhagic stroke; the RRs associated with each individual NSAID were similar for intracerebral hemorrhage and ischemic stroke. ${ }^{14}$ This study also estimated SAH risk separately from the overall analysis, but only for all NSAIDs combined $(\mathrm{RR}=1.2,95 \%$ $\mathrm{CI}=0.7-2.1)^{14}$

\section{Subgroup analyses}

Only two studies estimated the effect of dose and duration (Table 3). ${ }^{13,18}$ One study reported a tendency to a higher risk of ischemic stroke with the high doses

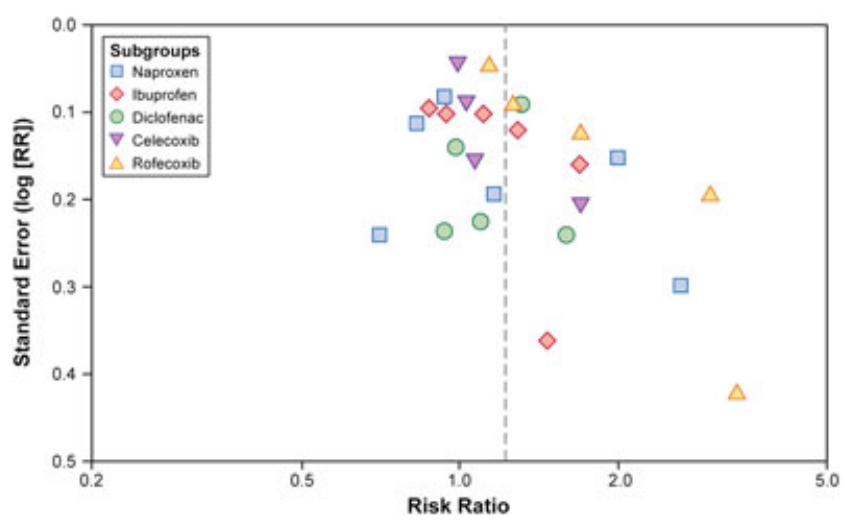

Figure 3. Funnel plot; RR of stroke for individual NSAIDs compared with NSAID nonuse ( $\mathrm{n}=6$ studies); $\mathrm{RR}$ is plotted on the horizontal axis and an estimate of its precision, SE (log RR), on the vertical axis of rofecoxib and etoricoxib but not celecoxib. ${ }^{13}$ The other study found no increased risk of stroke by dose for celecoxib, ibuprofen, naproxen, diclofenac, or indomethacin. ${ }^{18}$ For rofecoxib and valdecoxib, most stroke events were observed in the low-dose group.

Rofecoxib was associated with an increased risk of stroke in short- and long-term use, with higher risk associated with longer duration (Table 3). ${ }^{13}$ An increased risk of stroke associated with either valdecoxib or etoricoxib was observed in both short- and longterm users, using 90 days as the cutoff point. CIs included the null value (Table 3 ).

Two studies evaluated the effect in the new users of individual NSAIDs. ${ }^{18,19}$ One of the studies reported that the RR associated with each individual NSAID was slightly higher in new users than that obtained for all users. ${ }^{18}$

On the basis of two studies, the pooled RR for indomethacin use was 1.25 (95\% CI $=0.94-1.68)$ (data not shown). One study reported an increased risk of incident stroke associated with valdecoxib use $(\mathrm{RR}=1.41$, 95\% CI $=1.04-1.91)$ and another study with etoricoxib use $(\mathrm{RR}=2.38,95 \% \mathrm{CI}=1.10-5.13) .{ }^{13,18}$

No studies evaluated the risk of stroke associated with the concomitant use of NSAIDs and aspirin. One study reported that $39 \%$ of the cases of ischemic stroke and $22 \%$ of controls used low doses of aspirin. However, the investigators did not evaluate the effect by aspirin use ${ }^{13}$; the estimates were similarly elevated for rofecoxib and etoricoxib in patients with or without cardiovascular disease, atrial fibrillation, or hypertension.

\section{Sensitivity analysis}

We performed a subanalysis excluding the study that reported high RRs for naproxen and rofecoxib, but heterogeneity across studies was still present. ${ }^{15} \mathrm{We}$ also excluded the study that included only men and defined current exposure using a larger time window than that used in other studies. ${ }^{12}$ This exclusion reduced heterogeneity between studies and resulted in a pooled RR for rofecoxib of $1.32(95 \% \mathrm{CI}=1.07-1.62)$.

\section{DISCUSSION}

Our meta-analysis, including more than 14,375 stroke events, that is, 10,163 ischemic, 1403 haemorrhagic (intracerebral hemorrhage or SAH), and 273 of unspecified origin, supports an elevated risk of ischemic stroke associated with the current use of rofecoxib $(\mathrm{RR}=1.82,95 \% \quad \mathrm{CI}=1.09-3.04)$ and diclofenac $(\mathrm{RR}=1.20,95 \% \mathrm{CI}=0.99-1.45)$ of similar magnitude 


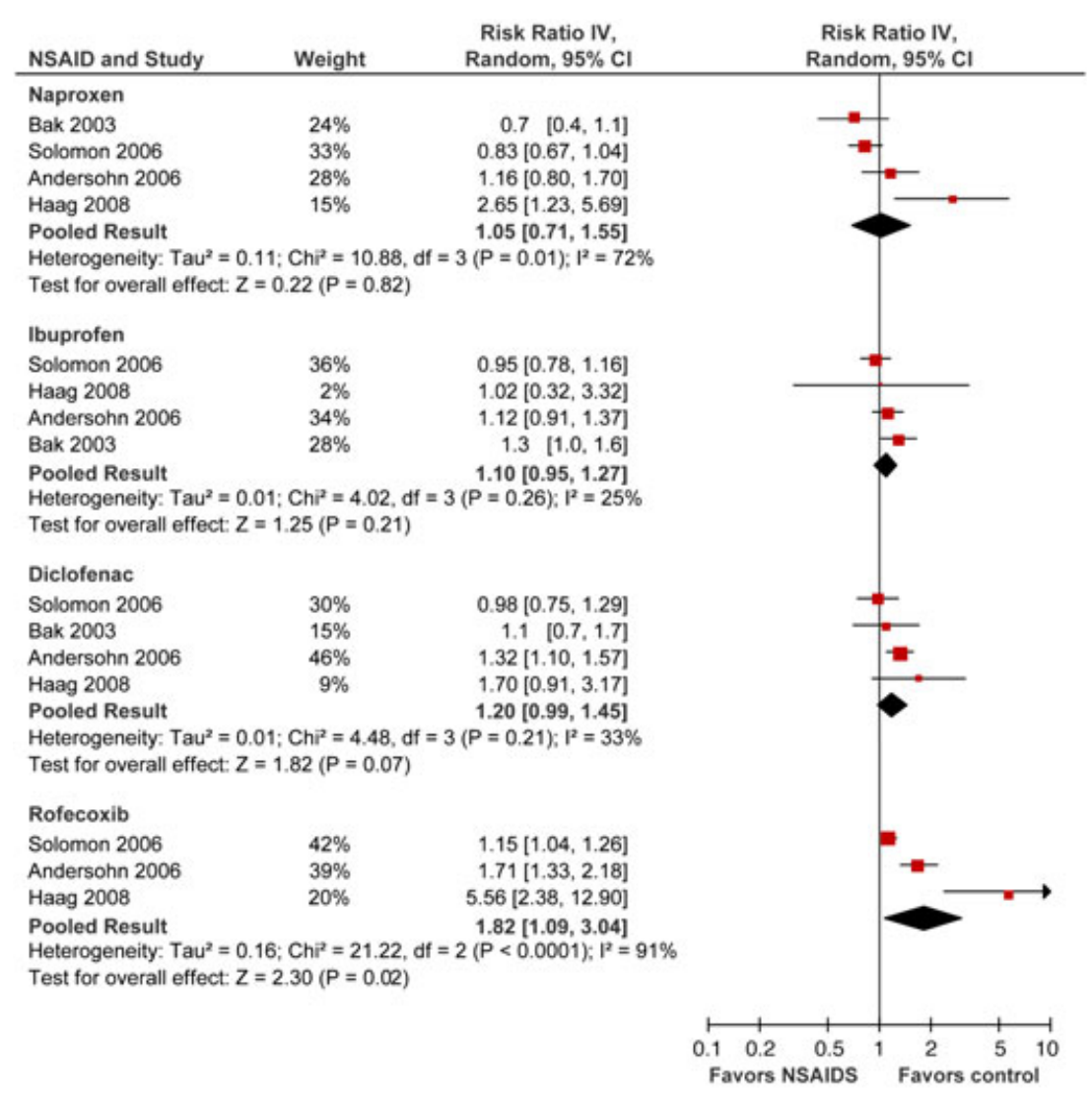

Figure 4. Forest plots of the risk of ischemic stroke associated with current use of naproxen, ibuprofen, diclofenac and rofecoxib relative to nonuse; results from published studies $(n=4)$ and pooled estimates by random effects

to that estimated in the prior meta-analyses of observational studies assessing the risk of AMI. ${ }^{6,8}$ Pooled RRs estimated for naproxen, ibuprofen, and celecoxib are also consistent with those previously estimated for AMI.

To our knowledge, this is the first meta-analysis of observational studies assessing the risk of stroke associated with use of individual NSAIDs compared with the nonuse of NSAIDs. Our meta-analysis was limited by the low number of published studies fulfilling the inclusion criteria. Only summary estimates for the most frequently used NSAIDs across the studied populations were obtained, and in some instances, these estimates might have been heavily influenced by the imprecise risk estimates in the individual studies.

Heterogeneity between studies was present for all individual NSAIDs except diclofenac. We evaluated the potential sources of heterogeneity instead of not pooling the data from these studies. Estimates were more homogenous between studies when restricting the analysis to incident events only or to ischemic subtype only. We calculated pooled effect estimates by both fixed and random effect models. Random effects models are recommended in the context of substantial heterogeneity; however, fixed effect models might be preferable when the number of studies is small.

The evidence on the effect of individual NSAIDs on the risk of stroke continues to be limited. The Adenomatous Polyp Prevention on Vioxx trial found a risk of ischemic stroke for rofecoxib twice that for placebo. ${ }^{2}$ A meta-analysis of randomized clinical trials reported a moderately increased risk of vascular events associated with selective COX-2 inhibitors, largely attributable to a twofold increased risk of AMI. The highdose regimens of some traditional NSAIDs such as diclofenac and ibuprofen were associated with similar excess risk of vascular events. However, the specific analysis for stroke failed to show an increased risk effect of either selective COX-2 inhibitors or traditional NSAIDs, likely because of the small number of events. In 121 clinical trials, 70 stroke events occurred in 18,490 person-years of selective COX-2 inhibitor 


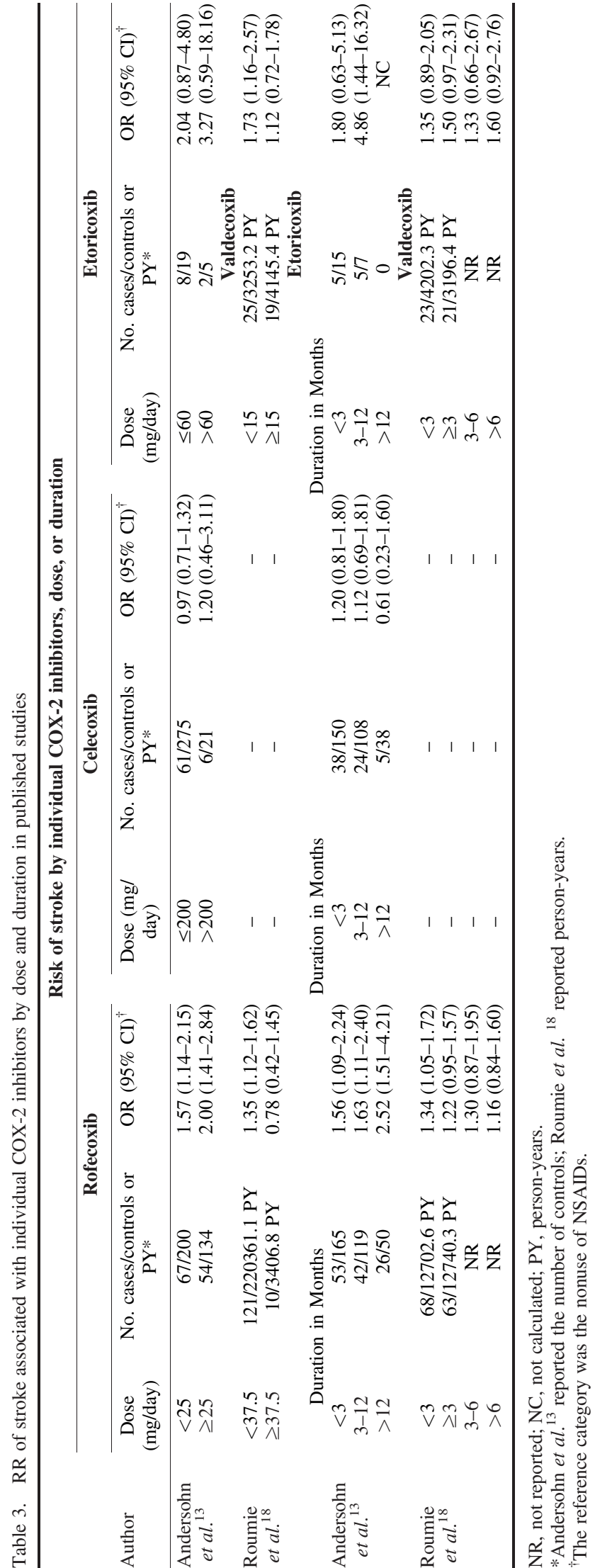

exposure compared with 53 events in 12,639 personyears in placebo. In fact, the upper limit of the $95 \%$ $\mathrm{CI}$ for the RR was 1.47 .

More recently, Trelle et al. ${ }^{22}$ have published a metaanalysis of large randomised controlled trials of NSAIDs. For stroke, 26 trials provided data with 337 observed events. Most of the individual NSAIDs evaluated presented a pooled rate ratio greater than 1 as compared with placebo. However, the $95 \%$ CIs of these estimates of risk were very wide, including the unity. Interestingly, ibuprofen, diclofenac, etoricoxib, and lumiracoxib showed the highest pooled estimates, whereas rofecoxib and celecoxib presented the smallest estimates of risk. The authors acknowledged, among some of the limitations, that the quality of the reported safety data from these trials was suboptimal.

The studies in our meta-analysis included broad study populations, although the US studies were all in elderly persons, and in one study, patients with baseline life-threatening conditions or neoplasms were excluded. ${ }^{18}$ Most the studies included both the new and the prevalent users of NSAIDs. The inclusion of prevalent users can introduce bias caused by the underascertainment of events that occur after the start of the therapy but before the start of the study (which may lead to depletion of susceptibles) and by the inability to control for risk factors that may be modified by the study drugs. ${ }^{23,24}$ Two studies provided RRs in new users ${ }^{18,19}$; one of them reporting a higher RR of stroke in new users than in all users. ${ }^{18}$

Potential biases secondary to the modification of risk factors by the study drugs can be avoided by measuring these factors at the beginning of the therapy when they are not influenced by the treatment. Hypertension can be considered a causal intermediate factor because it can be an effect of NSAIDs and is a strong risk factor for stroke. Thus, adjusting for hypertension or for the use of concurrent hypertensive medications during the follow-up could result in the underestimation of the RR. ${ }^{25}$ Most of the studies adjusted for hypertension only at baseline. Two studies conducted analysis stratified by the presence or absence of hypertension, but no effect modification was observed. ${ }^{13,19}$

The incomplete ascertainment of cases might potentially affect the results of these studies, but this misclassification should be nondifferential if unrelated with the exposure to NSAIDs. Most studies evaluated both hemorrhagic and ischemic stroke, but separate results were available only for ischemic stroke. Stroke is a complex condition, with different pathophysiology for and multiple etiologies within each subtype. In the studies that performed validation, the positive 
predictive value for overall stroke tended to be high (97\%). Assessing incident cases might be an issue without performing complete validation ${ }^{26}$; however, a major challenge is to differentiate between hemorrhagic and ischemic stroke, which is important for NSAID safety studies because the mechanism for each condition is different.

It has been reported that the extent of COX-2dependent prostacyclin inhibition may represent an independent key determinant of the increased thrombotic risk with NSAIDs in the presence of an insufficient reduction of platelet COX-1 activity $(<95 \%)$ to inhibit platelet function. ${ }^{11}$ Individual NSAIDs with a degree of COX-2 inhibition less than 90\% at therapeutic concentrations (ibuprofen, meloxicam, celecoxib, and etoricoxib) have been associated with an RR of AMI of 1.18 (95\% CI $=1.02-1.38)$, whereas those with greater COX-2 inhibition (rofecoxib, diclofenac, indomethacin, and piroxicam) had an RR of $1.60(95 \% \mathrm{CI}=1.41-1.81){ }^{27}$ The pooled estimates on the risk of stroke from our meta-analysis across the individual NSAIDs evaluated are consistent with this pattern.

Because NSAIDs are a chemically heterogeneous group of agents, the use of each compound might also translate into a differential effect not only on the risk of thrombotic events but also on hemorrhagic events. Recently, a study reported separately results on the risk of ischemic and hemorrhagic stroke. ${ }^{28}$ However, this study did not meet our inclusion criteria.

Recall bias is not an issue in any of the included studies because all assessed exposure by electronic prescriptions. No study captured the use of over-thecounter drugs; therefore, the effect of aspirin used for cardioprotection on the risk of stroke is not available. All of the studies described the baseline characteristic and risk profiles of the users of each individual NSAID. One study used disease risk scores to summarize potential confounders into a single multivariable model that predicts the risk of disease on the basis of measured covariates. ${ }^{29}$ In addition to age and sex, the most frequently considered covariates were transient ischemic attack, atrial fibrillation, heart failure, myocardial infarction, coronary heart disease, hypercholesterolemia, hypertension, diabetes, and arthritis.

There is still debate on whether the reduction of stroke incidence associated with statin use is due to the cholesterol-reduction effect only or to the pleiotropic effects of statins also, such as improved endothelial function, decreased platelet aggregability, and reduced vascular inflammation. Lipid-lowering drugs were considered in all of the studies except one that adjusted the individual's levels of total serum cholesterol. ${ }^{15}$
Residual confounding might be an issue in those studies that failed to systematically record some factors such as smoking, physical activity, socioeconomic status, or body mass index. In one of the studies, the users of COX-2 inhibitors were more likely than the nonusers to have a lower educational attainment, obesity, and currently smoke in a separate survey performed on the members of the source population. ${ }^{9}$ Investigators calculated that these differences resulted in a $2 \%$ bias away from the null. Therefore, although all of the studies in this meta-analysis might be affected by unmeasured confounding and by some misclassification of over-the-counter NSAID use, the bias was likely not differential between individual drugs.

Bias by contraindication to cardiovascular highrisk patients would be relatively minor because none of the studies included time after the withdrawal of rofecoxib.

Protopathic bias may occur if the exposure to the drug of interest started, stopped, or changed because of an unrecognized initial manifestation of the disease under study. ${ }^{30}$ One study reported the presence of protopathic bias in patients with stroke to be more prominent in those with SAH. In these patients, sentinel headache triggered the prescription of NSAIDs. ${ }^{14}$ None of the studies assessed migraine as potential confounder. ${ }^{31}$

One of the potential sources of heterogeneity can be the patterns of use of each individual NSAIDs across the study populations. In studies performed in the United States, diclofenac was less frequently used than in studies performed in European populations. This might have resulted in some underestimation of the risk of stroke associated with diclofenac use because of the imprecise estimates of risk obtained from these studies. In addition, we were not able to estimate pooled RRs stratified by dose and duration. Results provided in individual studies were limited by the low numbers of subjects exposed to high doses. Information from the few individual studies assessing the duration of use suggested that the risk of stroke associated with rofecoxib is present from the beginning of the therapy and might be higher with durations longer than 12 months. ${ }^{13}$

In conclusion, results from observational studies in this systematic review suggest a variability of effect on the risk of ischemic stroke across individual NSAIDs. Rofecoxib and diclofenac were associated with an increased risk of ischemic stroke compared with the nonuse of NSAID. Additional studies are needed to evaluate the risk of stroke associated with individual NSAIDs by dose, duration, concomitant use of aspirin, and separately for each stroke subtype and to address the potential for residual confounding. 


\section{KEY POINTS}

- To the best of our knowledge, this is the first meta-analysis of published results from observational studies on the risk of stroke associated with the use of NSAIDs.

- Our results suggest a variability of effect on the risk of ischemic stroke across individual NSAIDs; rofecoxib and diclofenac were associated with an increased risk compared with the nonuse of NSAID.

- Additional studies are needed to evaluate the risk of stroke associated with individual NSAIDs by dose, duration, and concomitant use of aspirin and separately for each stroke subtype and to address the potential for residual confounding.

\section{CONFLICT OF INTEREST}

JC, NRG, CVL, BC, and SPG are employees of RTIHS. JC, NRG, and SPG conducted research activities funded by Helsinn Healthcare S.A., a manufacturer of NSAIDs. SPG and CVL conducted research activities funded by NicOx S.A., a developer of NSAIDs. SPG has served on advisory boards, provided consultation to pharmaceutical companies including NSAID manufacturers/developers such as Helsinn and NicOx S.A, and is member of the scientific committee of a study on the safety of NSAIDs sponsored by Dundee University. Helsinn and $\mathrm{NicOx}$ were not involved in this study.

MS is coordinating the FP-7-funded SOS project. She is heading a research group at the Erasmus University that occasionally does drug safety studies for pharmaceutical companies such as AstraZeneca, Pfizer, EliLilly, and Novartis, and she has received unrestricted research grant support from AstraZeneca. Most of these companies produce NSAIDs, but they were not involved in this study.

AP is involved in a research funded by the European Commission via FP-7 call for projects.

LS declares no conflict of interest.

\section{ACKNOWLEDGEMENTS}

This work was supported by the European Community's Seventh Framework Programme under grant agreement number [223495] — the SOS project. The authors thank Ceri Hirst and Miny Samuel for their contributions in planning the literature review, Federica Nicotra for developing the database for the collection of abstracted data, Mendel Haag for the review and thoughtful comments during the research and final manuscript preparation, and Carla Franzoni for her support in managing the project.

\section{REFERENCES}

1. Bombardier C, Laine L, Reicin A, et al. Comparison of upper gastrointestinal toxicity of rofecoxib and naproxen in patients with rheumatoid arthritis. VIGOR Study Group. N Engl J Med 2000; 343: 1520-1528, 1522 p following 1528.

2. Bresalier RS, Sandler RS, Quan H, et al. Cardiovascular events associated with rofecoxib in a colorectal adenoma chemoprevention trial. N Engl J Med 2005; 352: 1092-1102. DOI: 10.1056/NEJMoa050493

3. Solomon SD, McMurray JJ, Pfeffer MA, et al. Cardiovascular risk associated with celecoxib in a clinical trial for colorectal adenoma prevention. $N$ Engl $J$ Med 2005; 352: 1071-1080. DOI: 10.1056/NEJMoa050405

4. Ott E, Nussmeier NA, Duke PC, et al. Efficacy and safety of the cyclooxygenase 2 inhibitors parecoxib and valdecoxib in patients undergoing coronary artery bypass surgery. J Thorac Cardiovasc Surg 2003; 125: 1481-1492.

5. Cardiovascular and cerebrovascular events in the randomized, controlled Alzheimer's Disease Anti-Inflammatory Prevention Trial (ADAPT). PLoS Clin Trials 2006; 1: e33. DOI: 10.1371/journal.pctr.0010033

6. Hernández-Díaz S, Varas-Lorenzo C, García Rodríguez LA. Non-steroidal antiinflammatory drugs and the risk of acute myocardial infarction. Basic Clin Pharmacol Toxicol 2006; 98: 266-274. DOI: 10.1111/j.1742-7843.2006. pto_302.x

7. Kearney PM, Baigent C, Godwin J, et al. Do selective cyclo-oxygenase-2 inhibitors and traditional non-steroidal anti-inflammatory drugs increase the risk of atherothrombosis? Meta-analysis of randomised trials. BMJ 2006; 332: 1302-1308. DOI: 10.1136/bmj.332.7553.1302

8. McGettigan P, Henry D. Cardiovascular risk and inhibition of cyclooxygenase: a systematic review of the observational studies of selective and nonselective inhibitors of cyclooxygenase 2. JAMA 2006; 296: 1633-1644. DOI: 10.1001/ jama.296.13.jrv60011

9. Scott PA, Kingsley GH, Smith CM, et al. Non-steroidal anti-inflammatory drugs and myocardial infarctions: comparative systematic review of evidence from observational studies and randomised controlled trials. Ann Rheum Dis 2007; 66: 1296-1304. DOI: 10.1136/ard.2006.068650

10. Capone ML, Tacconelli S, Rodriguez LG, et al. NSAIDs and cardiovascular disease: transducing human pharmacology results into clinical read-outs in the general population. Pharmacol Rep 2010; 62: 530-535.

11. Capone ML, Tacconelli S, Sciulli MG, et al. Clinical pharmacology of platelet, monocyte, and vascular cyclooxygenase inhibition by naproxen and low-dose aspirin in healthy subjects. Circulation 2004; 109: 1468-1471. DOI: 10.1161/01. CIR.0000124715.27937.78

12. Abraham NS, El-Serag HB, Hartman C, et al. Cyclooxygenase-2 selectivity of non-steroidal anti-inflammatory drugs and the risk of myocardial infarction and cerebrovascular accident. Aliment Pharmacol Ther 2007; 25: 913-924. DOI: 10.1111/j.1365-2036.2007.03292.x

13. Andersohn F, Schade R, Suissa S, et al. Cyclooxygenase-2 selective nonsteroidal anti-inflammatory drugs and the risk of ischemic stroke: a nested case-control study. Stroke 2006; 37: 1725-1730. DOI: 10.1161/01.STR. 0000226642.55207.94

14. Bak S, Andersen M, Tsiropoulos I, et al. Risk of stroke associated with nonsteroidal anti-inflammatory drugs: a nested case-control study. Stroke 2003; 34: 379-386.

15. Haag MD, Bos MJ, Hofman A, et al. Cyclooxygenase selectivity of nonsteroidal anti-inflammatory drugs and risk of stroke. Arch Intern Med 2008; 168: 1219-1224. DOI: 10.1001/archinte.168.11.1219

16. Huang WF, Hsiao FY, Tsai YW, et al. Cardiovascular events associated with long-term use of celecoxib, rofecoxib and meloxicam in Taiwan: an observational study. Drug Saf 2006; 29: 261-272.

17. Huang WF, Hsiao FY, Wen YW, et al. Cardiovascular events associated using four nonselective NSAIDs (etodolac, nabumetone, ibuprofen, or naproxen) versus a cyclooxygenase-2 inhibitor (celecoxib): a population-based analysis in Taiwanese adults. Clin Ther 2006; 28: 1827-1836. DOI: 10.1016/j. clinthera.2006.11.009

18. Roumie CL, Mitchel EF, Jr., Kaltenbach L, et al. Nonaspirin NSAIDs, cyclooxygenase 2 inhibitors, and the risk for stroke. Stroke 2008; 39: 2037-2045. DOI: 10.1161/STROKEAHA.107.508549

19. Solomon DH, Avorn J, Stürmer T, et al. Cardiovascular outcomes in new users of coxibs and nonsteroidal antiinflammatory drugs: high-risk subgroups and time course of risk. Arthritis Rheum 2006; 54: 1378-1389. DOI: 10.1002/art.21887

20. Wells GA, Shea B, O'Connell D, Peterson J, Welch V, Losos M, Tugwell P. The Newcastle-Ottawa Scale (NOS) for assessing the quailty of nonrandomised studies in meta-analyses. Ottawa, Ontario: Ottawa Health Research Institute 2010. Available at: http://www.ohri.ca/programs/clinical_epidemiology/oxford.htm. Accessed Mar 17, 2010.

21. DerSimonian R, Laird N. Meta-analysis in clinical trials. Control Clin Trials 1986; 7: 177-188. 
22. Trelle S, Reichenbach S, Wandel S, et al. Cardiovascular safety of non-steroidal anti-inflammatory drugs: network meta-analysis. BMJ 2011; 342: c7086. DOI: 10.1136/bmj.c7086

23. Ray WA. Evaluating medication effects outside of clinical trials: new-user designs. Am J Epidemiol 2003; 158: 915-920.

24. Suissa S, Garbe E. Primer: administrative health databases in observational studies of drug effects--advantages and disadvantages. Nat Clin Pract Rheumatol 2007; 3: 725-732. DOI: 10.1038/ncprheum0652

25. Greenland S. Introduction to regression models. Hagerstown, MD: LippincottRaven; 1998.

26. Roumie CL, Mitchel E, Gideon PS, et al. Validation of ICD-9 codes with a high positive predictive value for incident strokes resulting in hospitalization using Medicaid health data. Pharmacoepidemiol Drug Saf 2008; 17: 20-26. DOI: $10.1002 /$ pds. 1518

27. Garcia Rodriguez LA, Tacconelli S, Patrignani P. Role of dose potency in the prediction of risk of myocardial infarction associated with nonsteroidal anti-inflammatory drugs in the general population. J Am Coll Cardiol 2008; 52: 1628-1636. DOI: 10.1016/j.jacc.2008.08.041

28. Mangoni AA, Woodman RJ, Gilbert AL, et al. Use of non-steroidal anti-inflammatory drugs and risk of ischemic and hemorrhagic stroke in the Australian veteran community. Pharmacoepidemiol Drug Saf 2010. DOI: 10.1002/pds.1945

29. Arbogast PG, Ray WA. Use of disease risk scores in pharmacoepidemiologic studies. Stat Methods Med Res 2009; 18(1): 67-80. DOI: 10.1177/0962280208092347

30. Horwitz RI, Feinstein AR. Exclusion bias and the false relationship of reserpine and breast cancer. Arch Intern Med 1985; 145: 1873-1875.

31. Schürks M, Rist PM, Bigal ME, et al. Migraine and cardiovascular disease: systematic review and meta-analysis. BMJ 2009; 339: b3914.

\section{APPENDIX A}

\section{LITERATURE SEARCH STRATEGY FOR CARDIOVASCULAR ENDPOINTS}

PubMed was searched using the following groups of search terms, restricted to English-language publications and human subjects.

1. Pertinent, high-level medical subject headings (MeSH) and free-text terms related to cardiovascular events:

Stroke (hemorrhagic and ischemic), heart failure, myocardial infarction, and associated mortality

2. Pertinent MeSH and free-text terms to identify the relevant drugs.

3. Pertinent MesH and free-text terms related to cohort and case-control studies.

The PubMed search terms for cardiovascular endpoints are described in the strategy below.

1. stroke [Tiab] OR myocardial infarction [Tiab] OR heart failure [Tiab] OR cardiac failure [Tiab] OR left ventricular dysfunction [Tiab] OR acute coronary syndrome [Tiab] OR cerebrovascular disease [Tiab] OR coronary heart disease [Tiab] OR sudden cardiac death [Tiab] OR cardiovascular mortality [Tiab] OR coronary heart disease [Tiab] OR cardiorenal [Tiab] OR stroke [MeSH] OR myocardial infarction [MeSH] OR heart failure [MeSH]
OR ventricular dysfunction, left [MeSH] OR acute coronary syndrome $[\mathrm{MeSH}]$ OR death, sudden, cardiac [MeSH]

2. coxibs OR COX2 inhibitors OR cyclooxygenase 2 Inhibitors OR nsaids OR non-steroidal antiinflammatory OR "Anti-Inflammatory Agents, Non-Steroidal" [Mesh] OR phenylbutazone OR mofebutazone OR oxyphenbutazone OR clofezone OR kebuzone OR indometacin OR sulindac OR tolmetin OR zomepirac OR aceclofenac OR diclofenac OR alclofenac OR bumadizone OR etodolac OR lonazolac OR fentiazac OR acemetacin OR difenpiramide OR oxametacine OR proglumetacin OR ketorolac OR bufexamac OR indometacin, combinations OR diclofenac, combinations OR piroxicam OR tenoxicam OR droxicam OR lornoxicam OR meloxicam OR ibuprofen OR naproxen OR ketoprofen OR fenoprofen OR fenbufen OR benoxaprofen OR suprofen OR pirprofen OR flurbiprofen OR indoprofen OR tiaprofenic acid OR oxaprozin OR ibuproxam OR dexibuprofen OR flunoxaprofen OR alminoprofen OR dexketoprofen OR ibuprofen, combinations OR ketoprofen, combinations OR mefenamic acid OR tolfenamic acid OR flufenamic acid OR meclofenamic acid OR celecoxib OR rofecoxib OR valdecoxib OR parecoxib OR etoricoxib OR lumiracoxib OR nabumetone OR niflumic acid OR azapropazone OR glucosamine OR benzydamine OR glycosaminoglycan polysulfate OR proquazone OR orgotein OR nimesulide OR feprazone OR diacerein OR morniflumate OR tenidap OR oxaceprol OR chondroitin sulfate OR feprazone

3. cohort studies OR cohort OR epidemiologic methods OR case-control studies OR (case AND control) OR risk OR incidence

4. letter [Publication Type] OR editorial [Publication Type] OR comment [Publication Type]

5. \#1 AND \#2 AND \#3

6. \#5 NOT \#4

7. \#6 AND limits Entrez Date from 1990/01/01 to 2008/11/30; Humans, English

\section{APPENDIX B}

\section{QUALITY ASSESSMENT ACCORDING TO} THE NEWCASTLE-OTTAWA SCALE

Case-Control Studies

Among selected studies in the systematic review of stroke risk and NSAID use, there were two nested case-control studies for inclusion in the meta-analysis. 
The results of the quality evaluation according to the Newcastle-Ottawa Scale of these studies are presented in the table below for the selection, comparability, and exposure dimensions. The two studies scored the maximum numbers of starts for selection, comparability, and exposure.

\begin{tabular}{|c|c|c|c|c|}
\hline \multirow[b]{2}{*}{$\begin{array}{l}\text { Study's } \\
\text { first author }\end{array}$} & \multirow[b]{2}{*}{$\begin{array}{c}\text { Publication } \\
\text { year }\end{array}$} & \multicolumn{3}{|c|}{ Newcastle-Ottawa Scale Score } \\
\hline & & $\begin{array}{l}\text { Selection } \\
(\max .=4)\end{array}$ & $\begin{array}{l}\text { Comparability } \\
(\max .=2)\end{array}$ & $\begin{array}{l}\text { Exposure } \\
(\max .=3)\end{array}$ \\
\hline Bak & 2003 & $* * * *$ & ** & $* * *$ \\
\hline Andersohn & 2006 & $* * * *$ & ** & $* * *$ \\
\hline
\end{tabular}

\section{Cohort Studies}

A total of four cohort studies were selected for inclusion in the meta-analysis, and their quality was evaluated. The results of this evaluation are presented in the table below for the selection, comparability, and outcome dimensions. Two studies scored four stars, and three scored two stars for selection. For comparability and outcome, all four studies scored the maximum (two and three stars, respectively).

\begin{tabular}{lcccc}
\hline \multicolumn{4}{c}{ Newcastle-Ottawa Scale Score } \\
\hline Study's & $\begin{array}{c}\text { Publication } \\
\text { year }\end{array}$ & $\begin{array}{c}\text { Selection } \\
(\max .=4)\end{array}$ & $\begin{array}{c}\text { Comparability } \\
(\max .=2)\end{array}$ & $\begin{array}{c}\text { Outcome } \\
(\max .=3)\end{array}$ \\
Solomon & 2006 & $* * *$ & $* *$ & $* * *$ \\
Abraham & 2007 & $* * *$ & $* *$ & $* * *$ \\
Haag & 2008 & $* * * *$ & $* *$ & $* * *$ \\
Roumie & 2008 & $* * * *$ & $* *$ & $* * *$ \\
\hline
\end{tabular}

\section{APPENDIX C}

The SOS Project: list of research institutions

- Erasmus Universitair Medisch Centrum Rotterdam (EMC)

- Fundació IMIM (FIMIM)

- University of Nottingham (UNOTT)

- Università Degli Studi di Milano-Bicocca (UNIMIB)

- Research Triangle Institute (RTI-HS)

- Universitaet Bremen (Uni-HB)

- The Research Institute of the McGill University Health Centre (RI-MUHC)

- Azienda Ospedaliera di Padova (AOPD)

- PHARMO Coöperation UA (PHARMO)

- Université Victor Segalen Bordeaux II (UB2)

- Azienda Sanitaria Locale della provincia di Cremona (ASL/OSSIF) 